\title{
The Mean Value Theorem for Integrals Method for Estimating Two-Dimensional Renewal Functions
}

\author{
Leopoldus Ricky Sasongko', Bambang Susanto² \\ 1,2Program Studi Matematika, Universitas Kristen Satya Wacana, Indonesia \\ 1leopoldus.sasongko@uksw.edu, 2bambang.susanto@uksw.edu
}

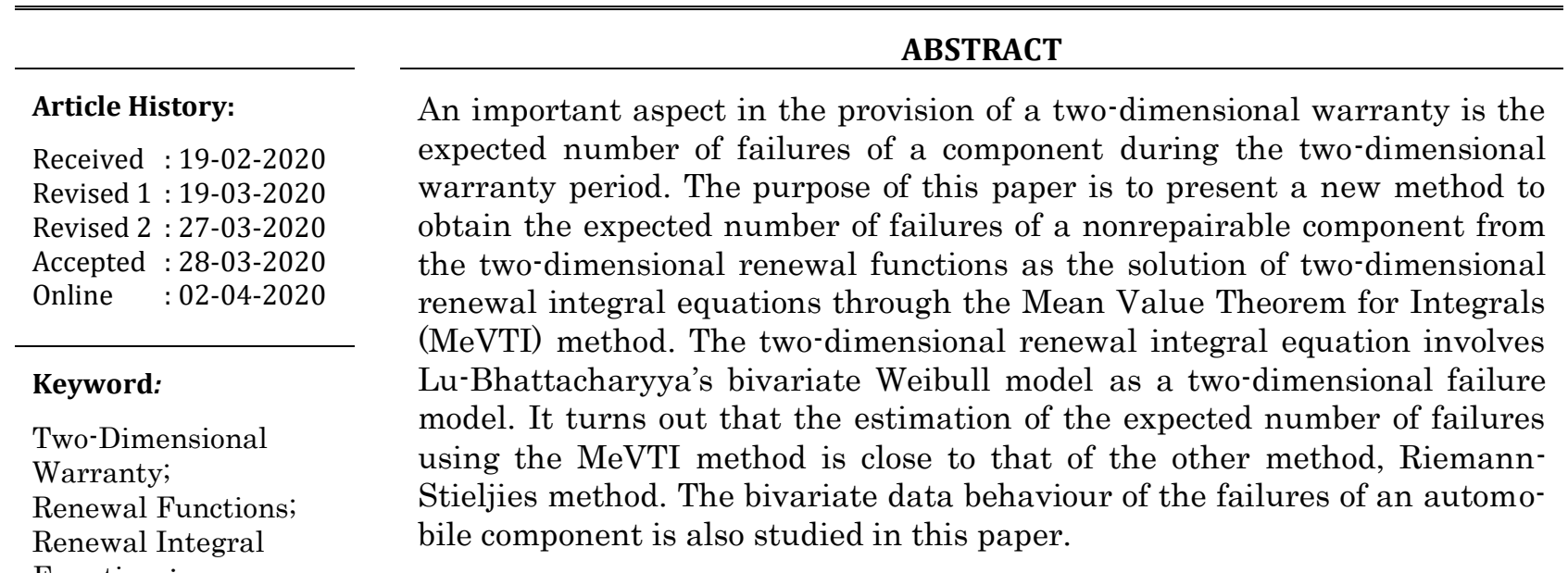

Equations;

Mean Value Theorem

for Integrals;

Bivariate Weibull

Model.

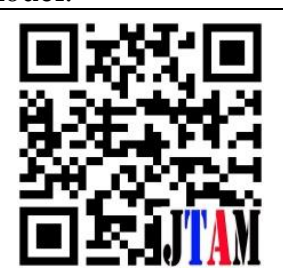

d. Crossref

https://doi.org/10.31764/itam.v4i1.1831

This is an open access article under the CC-BY-SA license

\section{A. INTRODUCTION}

We study an application in two-dimensional (2D) warranty modeling by considering a 2D free-replacement policy which is defined by a rectangle $[0, W) \times[0, U)$ in the 2D plane with the horizontal axis representing time and the vertical axis representing usage. The component that was covered by the warranty policy is nonrepairable and all failed components under warranty are replaced by new ones. Suppose $\left\{\left(X_{n}, Y_{n}\right), n \in \mathbb{N}\right\}$ denote the age and usage of the $n$th component at failure. By assuming that the time to replace is negligible and the failures are independent, this implies that a set of $\left\{\left(X_{n}, Y_{n}\right), n \in \mathbb{N}\right\}$ is a sequence of independent and identically distributed (i.i.d.) of nonnegative bivariate random variables with a common joint ditribution function $F(x, y)$ and number of failures over the rectangle $[0, x) \times[0, y)$ is given by a $2 \mathrm{D}$ renewal process, that is $N(x, y)$. Therefore, the expected number of failures over the 
rectangle $[0, x) \times[0, y)$ is given by $M(x, y)$ which is called the $2 \mathrm{D}$ renewal function (2D RNF) associated with $F(x, y)$.

Since $M(x, y)$ plays an important role in the 2D warranty modeling, it is really necessary to obtain the $M(x, y)$ analytically. However, even for the simplest forms of $F(x, y)$, obtaining $M(x, y)$ is difficult and complicated, or maybe impossible. Thus, computational techniques for estimating $M(x, y)$ are therefore required. One of computational techniques which have been widely used is Riemann-Stieljies (RS) method for estimating $M(x, y)$ which is expressed as the solution of the 2D renewal integral equation (2D RNIE).

We confine our attention to develop a numerical integration procedure that is based on Maghsoodloo-Helvaci's (2014) method where Sasongko \& Mahatma (2016) called it as the Mean Value Theorem for Integrals (MeVTI) method. We present a new numerical integration procedure that is based on the MeVTI method for estimating $M(x, y)$ which is expressed as the solution of the 2D RNIE. We also study the bivariate data behaviour of the failures of a component. We consider that the marginals of the data follow the Weibull distributions and the $M(x, y)$ is associated with Lu-Bhattacharyya's bivariate Weibull model. Then, the $M(x, y)$ is obtained by the estimation of the 2D RNIE using the MeVTI method.

\section{B. METHODS}

The 2D RNF plays an important role in the 2D warranty modeling. Blischke et al (2011) give basic explanation that we have to know about warranty analysis and modeling. Baik et al (2004) discuss about two-dimensional failure modeling, propose the character of the failures over a two-dimensional warranty region under minimal repair, and compare the use minimal repair strategy with replacement strategy. Baik et al (2006) give an erratum for their work in Baik et al (2004) at the character of the failures over a two-dimensional warranty region under minimal repair strategy but not under replacement strategy. Except in the minimal repair and replacement case, Banerjee \& Bhattacharjee (2012) discuss about an analysis of a twodimensional warranty with an imperfect repair strategy. Some literature of warranty application can be found such as in Chari et al (2013) which discuss the use of reconditioned products for unlimited free replacement warranty (FRW) policy, Januantoro \& Sarno (2018) use integer programming for optimizing cost and time of warranty process with repair and replacement strategy, and Rohman et al (2018) discuss two-dimensional warranty cost modeling with replacement strategy which involves copula function as a failure model.

We concern about obtaining a computational technique for estimating 2D RNF which is expressed as the solution of 2D RNIE. Generally, a numerical method that have been widely used for estimating 1D RNF or 2D RNF is a method based on integral Riemann-Stieljies. Pirade et al (2017) give basic explanation about the theory of integral Riemann-Stieljies. Two literatures those apply the integral Riemann-Stieljies for estimating the RNF which expressed as the RNIE are Xie (1989) for 1D RNIE case and Iskandar (1991) for 2D RNIE case. In this paper, the main method we concern to discuss for estimating the RNF is through the mean value theorem for integral method. Some literature discuss the basic theory and application of the mean value theorem for integral method such as Merikoski et al (2009), Mihai (2015), and Hutník \& Molnárová (2015). The application of the mean value theorem for integral in the warranty modeling have been used for estimating 1D RNIE in the Maghsoodloo \& Helvaci (2014) and Sasongko \& Mahatma (2016). Sasongko \& Mahatma (2016) called the mean value theorem for integrals as the MeVTI method. Several methods have been used to estimate or approximate the RNF. Kambo et al (2012) discuss and propose to approximate the RNF based only on first three moments. Some literature discuss the other approximation of the 2D RNF such as Hadji et al (2015), Arunachalam \& Calvache (2015), and Omey et al (2018). 
The 2D RNF which is expressed as the solution of the 2D RNIE is given by

$$
M(x, y)=F(x, y)+\int_{0}^{x} \int_{0}^{y} M(x-t, y-s) d F(t, s)
$$

Several steps to obtain our numerical integration for estimating (1) through MeVTI method are done by:

- Modifying variables $u=x-t$ and $v=y-s$,

- Considering that $F(x, y)=\int_{u=0}^{u=x} \int_{v=0}^{v=y} d F(x-u, y-v)$ and $\int_{0}^{x} \int_{0}^{y} M(x-t, y-s) d F(t, s)=\int_{u=0}^{u=x} \int_{u=0}^{v=y} M(u, v) d F(x-u, y-v)$ to the (1),

- Obtaining a new expression $M(x, y)=\int_{0}^{x} \int_{0}^{y}[1+M(u, v)] d F(x-u, y-v)$,

- Discretizing $[0, x) \times[0, y)$ into $n \times m$ rectangles that have equal area, they are $\left[x_{i-1}, x_{i}\right) \times\left[y_{j-1}, y_{j}\right)$ with $x_{i}=i \Delta x$ and $y_{j}=j \Delta y$ where $\Delta x=x / n$ and $\Delta y=y / m$,

- Considering that $M(x, y)=\sum_{i=1}^{n} \sum_{j=1}^{m}\left[\int_{x_{i-1}}^{x_{i}} \int_{y_{j-1}}^{y_{j}}[1+M(u, v)] d F(x-u, y-v)\right]$,

- Applying MeVTI on the point $\left(x_{i-1}, y_{j-1}\right)$ at every rectangles $\left[x_{i-1}, x_{i}\right) \times\left[y_{j-1}, y_{j}\right)$ to obtain the estimation of $M(x, y)$, that is given by

$$
\begin{aligned}
\widehat{M}(x, y) & =\sum_{i=1}^{n} \sum_{j=1}^{m}\left[\left[1+\widehat{M}\left(x_{i-1}, y_{j-1}\right)\right] \int_{x_{i-1}}^{x_{i}} \int_{y_{j-1}}^{y_{j}} d F(x-u, y-v)\right] \\
& =\sum_{i=1}^{n} \sum_{j=1}^{m}\left[\left[1+\widehat{M}\left(x_{i-1}, y_{j-1}\right)\right] \Delta_{x_{i-1}}^{x_{i}} \Delta_{y_{j-1}}^{y_{j}} F(x-u, y-v)\right]
\end{aligned}
$$

- Considering $\widehat{M}\left(x_{0}, y_{0}\right)=\widehat{M}(0,0)=M(0,0)=0$ and $\widehat{M}\left(x_{i}, 0\right)=\widehat{M}\left(0, y_{j}\right)=0$,

- Considering that $\widehat{M}(x, y)$ can be obtained forward recursively by

$$
\widehat{M}\left(x_{p}, y_{q}\right)=\sum_{i=1}^{p} \sum_{j=1}^{q}\left[\left(1+\widehat{M}\left(x_{i-1}, y_{j-1}\right)\right) \Delta_{x_{i-1}}^{x_{i}} \Delta_{y_{j-1}}^{y_{j}} F\left(x_{p}-u, y_{q}-v\right)\right]
$$

where $p=1,2,3, \ldots, n$ and $q=1,2,3, \ldots, m$.

\section{Lu-Bhattacharyya's bivariate Weibull model}

Lu \& Bhattacharyya (1990) propose a bivariate Weibull failure model which is given by the realibility function

$$
\bar{F}_{L B, \delta}(x, y)=\exp \left(-\left[\left(\frac{x}{\alpha_{1}}\right)^{\frac{\beta_{1}}{\delta}}+\left(\frac{y}{\alpha_{2}}\right)^{\frac{\beta_{2}}{\delta}}\right]^{\delta}\right)
$$

Thus, the corresponding bivariate distribution function is given by

$$
F_{L B, \delta}(x, y)=\exp \left(-\left[\left(\frac{x}{\alpha_{1}}\right)^{\frac{\beta_{1}}{\delta}}+\left(\frac{y}{\alpha_{2}}\right)^{\frac{\beta_{2}}{\delta}}\right]^{\delta}\right)+\exp \left[-\left(\frac{x}{\alpha_{1}}\right)^{\beta_{1}}\right]+\exp \left[-\left(\frac{y}{\alpha_{2}}\right)^{\beta_{2}}\right]-1
$$

where $\delta$ is the parameter dependence and $0<\delta \leq 1$. Note that (5) has marginals which are $X_{1} \sim F_{1}(x)=1-\exp \left[-\left(\frac{x}{\alpha_{1}}\right)^{\beta_{1}}\right]$ and $Y_{1} \sim F_{2}(y)=1-\exp \left[-\left(\frac{y}{\alpha_{2}}\right)^{\beta_{2}}\right]$, univariate Weibull. Baik et al (2004) and Lu \& Bhattacharyya (1990) note that $E\left[Y_{1} \mid X_{1}=x\right]$ increases with $x$. 


\section{Estimating the 2D RNF associated with Lu-Bhattacharyya's bivariate Weibull model}

We adopt an example which is found in Baik et al (2004). Let Lu-Bhattacharyya's bivariate Weibull model parameters be as follows:

$$
\alpha_{1}=2, \quad \beta_{1}=3, \quad \alpha_{2}=1.5, \quad \beta_{2}=2, \quad \delta=0.5
$$

The units for age and usage are years and $10^{4} \mathrm{~km}$, respectively. Then, the estimation of the 2D RNF associated with the model through MeVTI method or $\widehat{M}_{\mathrm{LB}, \delta}(W, U)$ at several rectangles $[0, W) \times[0, U)$ are shown in Table 1 . The numbers in the inside of [] are the results of the 2D RNF associated with the model through RS method which can be found in Baik et al (2004).

Table 1. The Estimation of the 2D RNF associated with the Lu-Bhattacharyya's bivariate Weibull

\begin{tabular}{|c|c|c|c|c|c|c|}
\cline { 3 - 7 } \multicolumn{2}{c|}{} & \multicolumn{5}{c|}{ Usage U (10000 km) } \\
\cline { 2 - 7 } \multicolumn{2}{c|}{} & $\mathbf{1}$ & $\mathbf{3}$ & $\mathbf{5}$ & $\mathbf{8}$ & $\mathbf{1 0}$ \\
\hline \multirow{4}{*}{$\begin{array}{c}\text { Age } W \\
\text { (years) }\end{array}$} & 1 & $0.095[0.095]$ & $0.298[0.301]$ & $0.324[0.328]$ & $0.326[0.330]$ & $0.326[0.330]$ \\
\cline { 2 - 7 } & 2 & $0.105[0.105]$ & $0.583[0.588]$ & $0.789[0.802]$ & $0.825[0.840]$ & $0.826[0.841]$ \\
\cline { 2 - 7 } & 3 & $0.106[0.106]$ & $0.698[0.704]$ & $1.164[1.181]$ & $1.347[1.374]$ & $1.359[1.388]$ \\
\cline { 2 - 7 } & 4 & $0.107[0.107]$ & $0.733[0.740]$ & $1.369[1.387]$ & $1.820[1.855]$ & $1.886[1.927]$ \\
\hline
\end{tabular}

\section{RESULT AND DISCUSSION}

\section{Data Set and Analysis}

We deal with a case study based on secondary data found in. Blischke et al (2011) give a part of warranty claims data for an automobile component (20 observations out of 498). We assume the component is nonrepairable. We also consider to assume failure modes, type of automobile used the component, and auto-used zone/region are negligible because of no detailed information about those (those are only shown in codes). Thus, we only use data consisted age in days and used KM at failure. The data have been scaled into the units for age and usage are months and $10^{4} \mathrm{~km}$, respectively. The data are shown in Table 2. Table 3 and Figure 1 show the preliminary analysis of the data.

We consider to estimate marginal age in months $\left(X_{1}\right)$ and usage per $10^{4} \mathrm{~km}\left(Y_{1}\right)$ to the univariate Weibull distributions. Figure 2 gives Weibull probability plot for each marginals. Table 4 gives the estimation of the parameters and the goodness of fit statistics.

Then, we consider to estimate the bivariate distribution of the bivariate data with the LuBhattacharyya's bivariate Weibull distribution. We use the vector generalized linier models (VGLM) method to estimate the parameter $\delta$ of the Lu-Bhattacharyya's bivariate Weibull distribution. The goodness of fit statistic of the estimation of the Lu-Bhattacharyya's bivariate Weibull distribution is done through the statistic Cramér-von Mises $\left(S_{N}\right)$ based on parametric bootstrap. The estimator of parameter and the goodness of fit statistic were given in Table 5.

Table 2. Failure data for an automobile component

\begin{tabular}{|c|c|c|c|c|c|}
\hline No. & $\begin{array}{c}\text { Age } \\
\text { (months) }\end{array}$ & $\begin{array}{c}\text { Usage } \\
\left(10^{4} \mathrm{~km}\right)\end{array}$ & No. & $\begin{array}{c}\text { Age } \\
\text { (months) }\end{array}$ & $\begin{array}{c}\text { Usage } \\
\left(10^{4} \mathbf{k m}\right)\end{array}$ \\
\hline 1 & 4.5333 & 3.6487 & 11 & 5.6333 & 1.8175 \\
\hline 2 & 3.4667 & 0.2381 & 12 & 5.6333 & 1.8106 \\
\hline 3 & 3.3000 & 1.4507 & 13 & 12.1333 & 2.7008 \\
\hline 4 & 3.1333 & 0.7377 & 14 & 2.6000 & 1.1600 \\
\hline 5 & 3.1333 & 1.0790 & 15 & 2.6000 & 0.7900 \\
\hline 6 & 5.2000 & 4.7312 & 16 & 2.5667 & 1.7620 \\
\hline 7 & 9.8333 & 5.6943 & 17 & 0.5333 & 0.7762 \\
\hline 8 & 10.0000 & 4.5292 & 18 & 0.5000 & 3.9487 \\
\hline 9 & 1.8000 & 0.5187 & 19 & 1.4667 & 0.6420 \\
\hline 10 & 2.7333 & 0.4512 & 20 & 6.1667 & 4.5121 \\
\hline
\end{tabular}


Table 3. Summary statistics for the variables age and usage for failed components

\begin{tabular}{lcccccccrr}
\hline Statistics & Mean & \multicolumn{1}{c}{ Var. } & Min & $\boldsymbol{Q}_{\mathbf{1}}$ & $\boldsymbol{Q}_{\mathbf{2}}$ & $\boldsymbol{Q}_{\mathbf{3}}$ & Max & Skewness & Kurtosis \\
\hline Age (months) & 4.348 & 10.047 & 0.500 & 2.575 & 3.217 & 5.633 & 12.133 & 1.190 & 3.900 \\
\hline Usage $\left(10^{4} \mathrm{~km}\right)$ & 2.150 & 2.971 & 0.238 & 0.747 & 1.606 & 3.874 & 5.694 & 0.770 & 3.850 \\
\hline
\end{tabular}

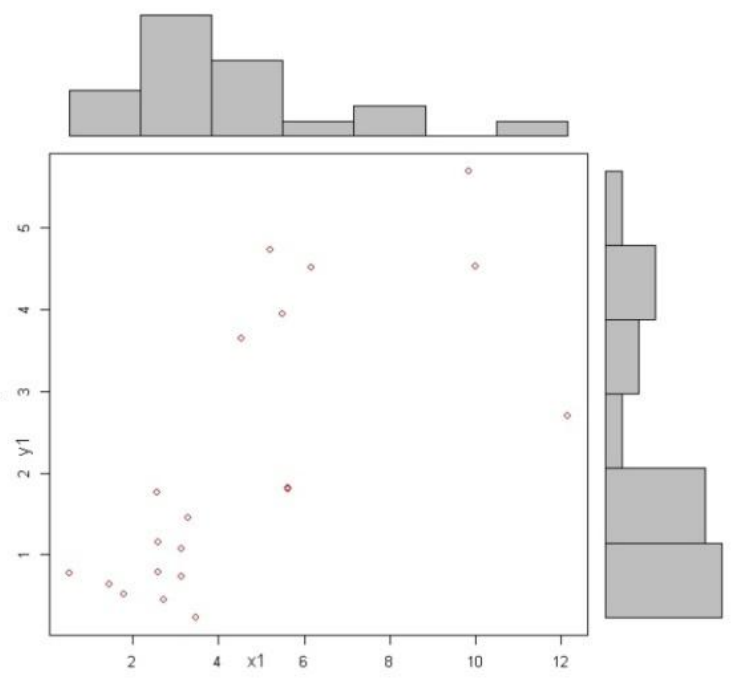

Figure 1. Marginal plot of age (months) versus usage $(10000 \mathrm{~km})$

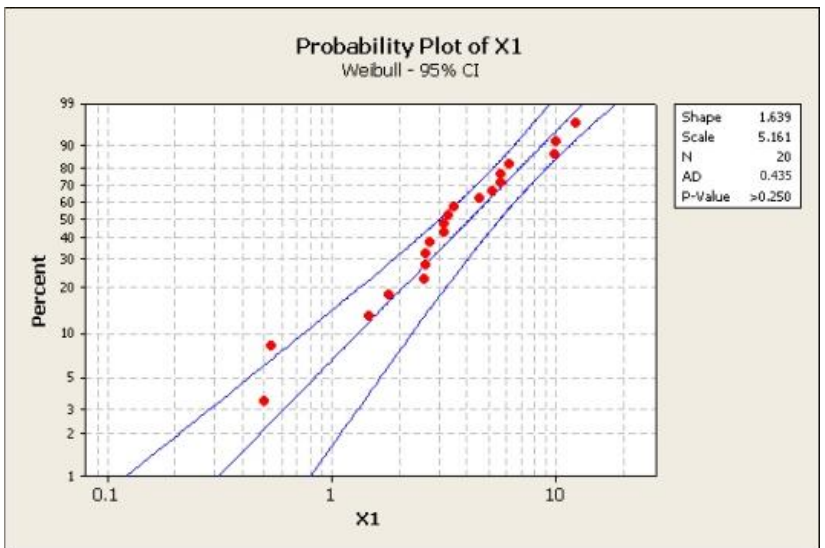

(a)

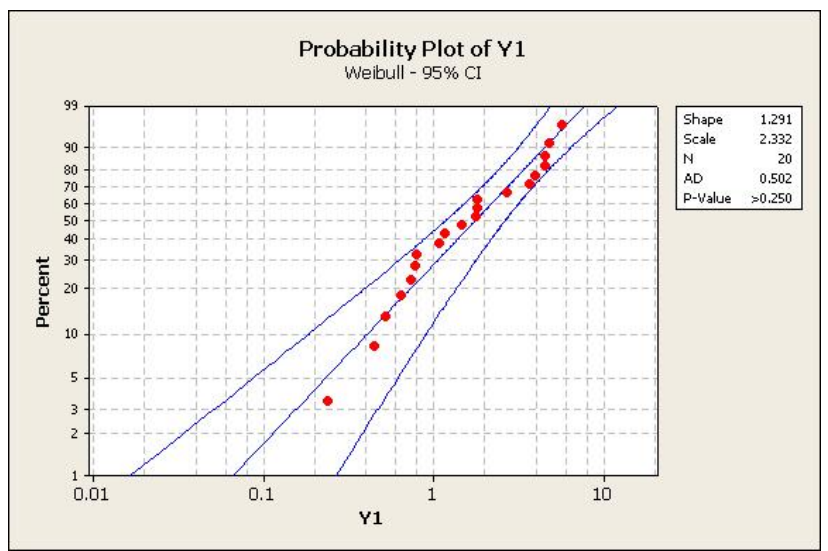

(b)

Figure 2. Weibull Probability Plot for (a) marginal $X_{1}$ and (b) marginal $Y_{1}$

Table 4. Parameters and goodness of fit statistics for marginal $X_{1}$ and marginal $Y_{1}$

\begin{tabular}{|c|c|c|c|c|c|c|c|}
\hline \multirow{3}{*}{$i$} & \multirow{3}{*}{$\begin{array}{l}\text { Mar- } \\
\text { ginal } \\
\text { Data }\end{array}$} & \multicolumn{4}{|c|}{ Univariate Weibull Distributions } & \multicolumn{2}{|c|}{ Goodness of Fit Statistics } \\
\hline & & \multicolumn{2}{|c|}{$\begin{array}{l}\text { Estimator of the } \\
\text { Parameters }\end{array}$} & \multicolumn{2}{|c|}{$\begin{array}{l}\text { Standard Error of } \\
\text { the estimators }\end{array}$} & \multirow{2}{*}{$\begin{array}{c}\begin{array}{c}\text { Kolmogorov- } \\
\text { Smirnov }\end{array} \\
\text { Stat. KS (p-value })\end{array}$} & \multirow{2}{*}{$\begin{array}{c}\begin{array}{c}\text { Anderson- } \\
\text { Darling }\end{array} \\
\text { Stat. AD (p-value })\end{array}$} \\
\hline & & $\widehat{\boldsymbol{\alpha}}_{i}$ & $\widehat{\boldsymbol{\beta}}_{\boldsymbol{i}}$ & $S E\left(\widehat{\boldsymbol{\alpha}}_{i}\right)$ & $S E\left(\widehat{\boldsymbol{\beta}}_{i}\right)$ & & \\
\hline 1 & $X_{1}$ & 5.1614 & 1.6385 & 0.7447 & 0.2776 & $0.1440 \quad(0.8015)$ & $0.4348(0.8119)$ \\
\hline 2 & $Y_{1}$ & 2.3324 & 1.2910 & 0.4272 & 0.2278 & $0.1345 \quad(0.8163)$ & $0.5024 \quad(0.7423)$ \\
\hline
\end{tabular}

Table 5. Parameter and goodness of fit statistic for the bivariate data

\begin{tabular}{cccc}
\hline \multirow{2}{*}{$\begin{array}{c}\text { Bivariate } \\
\text { Data }\end{array}$} & \multicolumn{2}{c}{$\begin{array}{c}\text { Lu-Bhattacharyya Bivariate } \\
\text { Weibull Distributions }\end{array}$} & Goodness of Fit Statistics \\
\cline { 2 - 4 } & $\begin{array}{c}\text { Estimator of the } \\
\text { Parameter }\end{array}$ & $\begin{array}{c}\text { Standard Error } \\
\text { of the estimator }\end{array}$ & $\begin{array}{c}\text { Cramér-von Mises based on } \\
\text { parametric bootstrap }\end{array}$ \\
\cline { 2 - 4 } & $\hat{\delta}$ & $S E(\hat{\delta})$ & Stat. $S_{N}(p$-value $)$ \\
\hline$\left(X_{1}, Y_{1}\right)$ & 0.4842 & 0.0690 & $0.0514(0.0674)$ \\
\hline
\end{tabular}




\section{Results}

We have the MeVTI method for estimating the 2D RNF which is expressed as the solution of the 2D RNIE in (3). We estimate the 2D RNF associated with Lu-Bhattacharyya's bivariate Weibull model where the parameters are $\alpha_{1}=2, \beta_{1}=3, \alpha_{2}=1.5, \beta_{2}=2$, and $\delta=0.5$. The estimation of the 2D RNF associated with the model through MeVTI method at several rectangles $[0, W) \times[0, U)$ are shown in Table 1 . The numbers in the inside of [] in Tabel 1 are the estimation of the 2D RNF associated with the same model through RS method. By considering the result in the Table 1, it turns out that the estimation of the 2D RNF through MeVTI method is close to the estimation of the 2D RNF through RS method. However, we do not have the exact estimation of the 2D RNF associated with the model. Thus, we can not compare which the better method is, about the accuracy of the methods.

We consider that the bivariate data have marginals which are univariate Weibull distributions, where the marginal $X_{1}$ has parameters $\hat{\alpha}_{1}=5.1614, \hat{\beta}_{1}=1.6385$, and the marginal $Y_{1}$ has parameters $\hat{\alpha}_{2}=2.3324, \hat{\beta}_{2}=1.2910$. By considering Tabel 5 , the bivariate data follow the Lu-Bhattacharyya's bivariate Weibull distribution with parameter $\hat{\delta}=0.4842$ at level of significancy is less than or equal 0.05 . Then, the 2D RNF associates with Lu-Bhattacharyya's bivariate Weibull model where the parameters are $\hat{\alpha}_{1}=5.1614, \hat{\beta}_{1}=1.6385, \hat{\alpha}_{2}=2.3324$, $\hat{\beta}_{2}=1.2910$, and $\hat{\delta}=0.4842$. The estimation of 2D RNF associated with the model through MeVTI method at several $[0, W) \times[0, U)$ are shown in Table 6 . However, at the level of significancy greater than 0.5 , we reject the model. Thus, other models are therefore required.

Table 6. The estimation of the $2 \mathrm{D}$ RNF associated with model of the bivariate data

\begin{tabular}{ccccccc} 
& & \multicolumn{5}{c}{ Usage $\boldsymbol{U}\left(\mathbf{1 0}^{\mathbf{4}} \mathbf{~ k m}\right)$} \\
\cline { 2 - 7 } & 1 & $\mathbf{1}$ & $\mathbf{2}$ & $\mathbf{3}$ & $\mathbf{4}$ & $\mathbf{5}$ \\
\hline \multirow{3}{*}{ Age $W$} & 3 & 0.2291 & 0.0656 & 0.0663 & 0.0665 & 0.0666 \\
\cline { 2 - 7 } (months) & 6 & 0.3034 & 0.6280 & 0.3569 & 0.3664 & 0.3699 \\
\cline { 2 - 7 } & 9 & 0.3147 & 0.7104 & 1.0651 & 1.3199 & 1.4730 \\
\cline { 2 - 7 } & 12 & 0.3166 & 0.7299 & 1.1487 & 1.5228 & 1.8140 \\
\hline
\end{tabular}

\section{CONCLUSION AND SUGGESTIONS}

We have provided a new numerical integration through the MeVTI method for estimating the 2D RNF. We have applied our method to estimate the 2D RNF which is associated with LuBhattacharyya's bivariate Weibull failure model. It turns out that the estimation of the 2D RNF through MeVTI method is close to the estimation of the 2D RNF through RS method. However, we do not have the exact estimation of the 2D RNF associated with the model. Thus, we can not compare which the better method is, about the accuracy of the methods.

We have studied and analyzed the bivariate data behaviour of the failures of an automobile component which is assumed as nonrepairable component. We have obtained that the data follow the Lu-Bhattacharyya's bivariate Weibull failure model at the level of significancy is less than or equal 0.05 and the estimation of the 2D RNF associated with the model. However, at level of significancy is greater than 0.5 , we reject the model. Thus, other models are therefore required.

\section{REFERENCES}

Arunachalam, V., \& Calvache, Á. (2015). Approximation of the bivariate renewal function. Communications in Statistics: Simulation and Computation, 44(1), 154-167. https://doi.org/10.1080/03610918.2013.770306 
Baik, J., Murthy, D. N. P., \& Jack, N. (2004). Two-dimensional failure modeling with minimal repair. Naval Research Logistics, 51(3), 345-362. https://doi.org/10.1002/nav.10120

Baik, J., Murthy, D. N. P., \& Jack, N. (2006). Erratum: Two-dimensional failure modeling with minimal repair which appeared in this journal of April 2004 (Naval Research Logistics 51:3 (345-362)). Naval Research Logistics, 53(1), 115-116. https://doi.org/10.1002/nav.20115

Banerjee, R., \& Bhattacharjee, M. C. (2012). Analysis of a Two-dimensional Warranty Servicing Strategy with an Imperfect Repair Option. 9(1), 23-33.

Blischke, W. R., Karim, M. R., \& Murthy, D. N. P. (2011). Warranty Data Collection and Analysis. In Springer Series in Reliability Engineering. https://doi.org/10.1007/978-1-4471-4588-2

Chari, N., Diallo, C., \& Venkatadri, U. (2013). Optimal unlimited free-replacement warranty strategy using reconditioned products. International Journal of Performability Engineering, 9(2), 191-200.

Hadji, E. M., Kambo, N. S., \& Rangan, A. (2015). Two-dimensional Renewal Function Approximation. Communications in Statistics - Theory and Methods, 44(15), 3107-3124. https://doi.org/10.1080/03610926.2013.815204

Hutník, O., \& Molnárová, J. (2015). On Flett's mean value theorem. Aequationes Mathematicae, 89(4), 1133-1165. https://doi.org/10.1007/s00010-014-0311-5

Iskandar, B. (1991). Two-dimensional renewal function solver. Research Report No. 4/91. Department of Mechanical Engineering. The University of Queensland. Brisbane. Australia.

Januantoro, A., \& Sarno, R. (2018). Repair and replacement strategy for optimizing cost and time of warranty process using integer programming. Telkomnika (Telecommunication Computing Electronics and Control), 16(6), 2683-2691. https://doi.org/10.12928/TELKOMNIKA.v16i6.10407

Kambo, N. S., Rangan, A., \& Hadji, E. M. (2012). Moments-based approximation to the renewal function. Communications in Statistics - Theory and Methods, 41(5), 851-868. https://doi.org/10.1080/03610926.2010.533231

Lu, J. C., \& Bhattacharyya, G. K. (1990). Some new constructions of bivariate Weibull models. In Annals of the Institute of Statistical Mathematics (Vol. 42, Issue 3, pp. 543-559). https://doi.org/10.1007/BF00049307

Maghsoodloo, S., \& Helvaci, D. (2014). Renewal and renewal-intensity functions with minimal repair. Journal of Quality and Reliability Engineering, 2014. https://doi.org/10.1155/2014/857437

Merikoski, J. K., Halmetoja, M., \& Tossavainen, T. (2009). Means and the mean value theorem. International Journal of Mathematical Education in Science and Technology, 40(6), 729-740. https://doi.org/10.1080/00207390902825328

Mihai, M. (2015). An Integral Mean Value Theorem concerning Two Continuous Functions and Its Stability. International Journal of Analysis, 2015(1), 1-4. https://doi.org/10.1155/2015/894625

Omey, E., Mitov, K., \& Vesilo, R. (2018). Approximations in bivariate renewal theory. Publications de l'Institut Mathematique, 104(118), 69-88. https://doi.org/10.2298/PIM18180690

Pirade, S., Manurung, T., \& Titaley, J. (2017). Integral Riemann-Stieltjes Pada Fungsi Bernilai Real. D'CARTESIAN, 6(1), 1. https://doi.org/10.35799/dc.6.1.2017.14987

Rohman, N., Mahatma, T., \& Sasongko, L. R. (2018). Pemodelan Biaya Garansi Dua Dimensi Polis FRW(Non-Renewing Free Replacement Warranty) dengan Strategi Penggantian untuk Oil Filter Mobil. D'CARTESIAN, 7(1), 1. https://doi.org/10.35799/dc.7.1.2018.19547

Sasongko, L., \& Mahatma, T. (2016). The Estimation of Renewal Functions Using the Mean Value Theorem for Integrals (MeVTI) Method. D'CARTESIAN, 5(2), 111. https://doi.org/10.35799/dc.5.2.2016.14984

Xie, M. (1989). On the solution of renewal-type integral equations. Communications in Statistics Simulation and Computation, 18(1), 281-293. https://doi.org/10.1080/03610918908812760 